\title{
Minimally Invasive Surgery for the Treatment of Hyperacusis
}

\author{
$*$ Herbert Silverstein, $*$ Rosemary Ojo, $*$ Julie Daugherty, $\nmid$ Ronen Nazarian, and $*$ Jack Wazen \\ *Ear Research Foundation, Sarasota, Florida; and †Osborne Head \& Neck Institute, Cedar Sinai Medical Center, \\ Los Angeles, California
}

Objective: To evaluate the efficacy of a minimally invasive surgical procedure in patients with severe hyperacusis.

Study Design: Prospective, longitudinal design.

Setting: Tertiary referral center.

Patients: Adult patients with history of severe hyperacusis. Intervention: Using a transcanal approach, the round and oval window was reinforced with temporalis fascia or tragal perichondrium in six subjects (nine ears) and was subdivided into two groups (unilateral or bilateral reinforcement procedure).

Main Outcome Measures: Pre- and postoperative noise tolerance was measured using uncomfortable loudness level (ULL) test scores. In addition, a self-report hyperacusis questionnaire (HQ) was used to assess hypersensitivity to sound before and after the intervention.

Results: Analysis of the data reveals improved postoperative mean ULL test scores of $14 \mathrm{~dB}$ (confidence interval [CI], $70-98 \mathrm{~dB}$ ) in the unilateral group. For the bilateral group, improved mean scores were $13 \mathrm{~dB}(\mathrm{CI}, 63-88 \mathrm{~dB})$ in the first ear and $8 \mathrm{~dB}(\mathrm{CI}, 71-86 \mathrm{~dB})$ for the second ear. Further, a negative linear trend was observed in the mean subjective scores for the HQ when both groups measures were analyzed together decreasing from a mean score of 32.0 (standard deviation $[\mathrm{SD}]=3.32$ ) preoperative to a mean score of 11.5 $(\mathrm{SD}=7.42)$ after surgery. Postoperatively, the patients reported no change in hearing and improved quality of life after the procedure.

Conclusion: The results suggest that reinforcement of the round and oval window with temporalis fascia or tragal perichondrium may offer significant benefit for individuals with severe hyperacusis that has not responded to traditional therapy. ULL scores and self-report measures postoperatively demonstrate improved noise tolerance, high patient satisfaction, and enhanced quality of life. Key Words: HyperacusisLoudness discomfort level-Oval window reinforcementRound window reinforcement.

Otol Neurotol 37:1482-1488, 2016.
Hyperacusis is an auditory phenomenon described by individuals as a reduction of normal tolerance for everyday sounds. Baguley and Andersson (1) clearly defined hyperacusis as an "experience of inordinate loudness to sound that is well tolerated by most people with an associated component of distress." Individuals with hyperacusis find certain sounds more difficult to listen to than others and may cause pain in their ears. Further, their quality of life is often diminished, because of anxiety and avoidance of noise related activities $(2,3)$. Previously published internet and general survey-based studies describe the prevalence of hyperacusis between 5.9 and $17.2 \%(4,5)$. Unfortunately, there is a paucity of epidemiological studies that estimate only the prevalence of hyperacusis. The majority of existing studies describe

Address correspondence and reprint requests to Herbert Silverstein, M.D., Ear Research Foundation, 1901 Floyd Street, Sarasota, FL 34239; E-mail: EarSinus@aol.com

The authors disclose no conflicts of interest.

This is an open-access article distributed under the terms of the Creative Commons Attribution-Non Commercial-No Derivatives License 4.0, where it is permissible to download and share the work provided it is properly cited. The work cannot be changed in any way or used commercially.

DOI: 10.1097/MAO.0000000000001214 the occurrence of hyperacusis in patients with tinnitus. The incidence of hyperacusis in individuals with tinnitus has been reported as high as 40 to $86 \%(6,7)$.

Jastreboff and Jastreboff (8) proposed a different taxonomy for decreased sound tolerance (DST); defining DST as the presence of negative reactions experienced as a result of exposure to sounds that would not evoke such aversive reactions in the average listener. They suggest DST can be subdivided into hyperacusis, misophonia, phonophobia, or a combination of these conditions (9). Furthermore, they characterize hyperacusis as a negative response to a sound dependent only on its physical characteristics, whereas misophonia is characterized in an individual as a negative reaction to a very distinct sound with a specific pattern and meaning. In addition, phonophobia is distinguished by a fear of sound, evoking behavioral changes such as avoidance of sound and psychological distress, even when anticipating exposure to sound.

The mechanism of hyperacusis is poorly understood, but has been related to acoustic overexposure resulting in increased gain within the central auditory pathways (10). Recruitment differs from the hyperacusis phenomenon. Recruitment is an abnormal physiologic response to sound that can be demonstrated with electrophysiologic 
recording and has been reported as abnormally rapid growth of loudness when sound intensity is increased in the damaged ear (11). While the etiology is often unknown, the most common underlying causes of hyperacusis reported in the literature include cochlear trauma, head injury, adverse medication reactions, hearing loss, aging, surgery, chronic ear infections, superior semicircular canal dehiscence (SSCD), and autoimmune disorders (12). A relationship between hyperacusis and perilymph fistula has been previously established in the literature $(13,14)$.

Currently, the treatment options for noise intolerance include avoidance of provocative stimuli, cognitive behavioral therapy (CBT), tinnitus retraining therapy, and hearing amplification, all with varied rates of efficacy (15). CBT has been shown to be effective when exposure to sounds is in a controlled and step-wise fashion, thereby reducing avoidance as well as audiological sensitivity. Unfortunately, there is limited published data on the benefit of CBT in patients with hyperacusis and further investigation is warranted (16).

There has been no corrective surgical procedure specifically for the treatment of hyperacusis. However, surgical intervention was found to improve hyperacusis in the patients with SSCD. Silverstein et al. (17) found round window reinforcement to be an effective and minimally invasive surgical option to reduce the symptoms of SSCD. Nikkar-Esfahani et al. (18) found an improvement in noise tolerance in patients with SSCD whose main complaint was hyperacusis and successfully underwent complete occlusion of round window niche. In a case report by Dang et al. (19), a patient with unilateral posterior and superior canal dehiscence underwent transmastoid plugging of both defects, resulting in complete resolution of hyperacusis. Silverstein et al. (20) reported the first successful surgical treatment of hyperacusis using temporalis fascia to reinforce the round and oval window. These observations led to the innovation of the present minimally invasive procedure for use in individuals with a chief complaint of hyperacusis without evidence of SSCD. The aim of this study is to evaluate the efficacy of this minimally invasive procedure in patients who suffer from intractable hyperacusis that have failed conventional therapy.

\section{MATERIALS AND METHODS}

A prospective, repeated-measure single-arm design was used for this study. Institutional Review Board (IRB) approval was obtained before the initiation of any study-related activities. Nine patients were enrolled between June 2014 and April 2016. The inclusion criteria were: 1) adults 18 years or older; 2) at least a 3-month history of hyperacusis symptoms before enrollment; 3 ) moderate to severe noise intolerance as determined by scores on the hyperacusis questionnaire (HQ) and uncomfortable loudness level (ULL) testing; and 4) no contraindication for surgical intervention under general anesthesia. Individuals with a history of Meniere's disease; active middle ear disease; women who were pregnant and individuals with a score of 11 or greater on the Hospital Anxiety and Depression Scale (HADS) were excluded from the study. Once informed consent was obtained, participants completed a comprehensive auditory evaluation including pure tone air and bone conduction thresholds, speech discrimination, and tympanometry. Psychoacoustic assessment was performed using a standardized protocol to determine the ULL's for each participant. Further, a self-report measure (HQ) was used to assess the participant's hypersensitivity to sound and the HADS was used to screen for anxiety or depression. Pre-operative evaluation also included a physical examination and computerized tomography (CT) of the temporal bone was obtained to rule out SSCD. Participants that met eligibility criteria then underwent the round window and oval window reinforcement procedure. Routine postoperative visits occurred at 1,4 , and 24 weeks after the intervention. Audiometric measures, ULL testing, and the HQ were obtained preoperatively and also at 4 and 24 weeks after the procedure. If the patient reported improvement after the first ear was operated but continued to have hyperacusis in the unoperated ear, surgery was performed 1 month after the first procedure.

\section{Hyperacusis Questionnaire}

The HQ used in this study is a non-validated English language adaptation of the Geräuschiberempfindlichkeit (GÜF), a German self-report measure for hypersensitivity to sound (14). It is composed of 15-items, evaluating three dimensions or subscales: cognitive behavior in relation to hyperacusis; somatic behavior or reaction linked to specific situation; and finally emotional reactions (21). Using a fourpoint Likert-type scale, respondents answer "no" (0 points), "yes, a little" (1 point), "yes, quite a lot ( 2 points), and "yes, a lot'" (3 points) for each item. The total possible score range is 0 to 45 , with higher scores indicating higher sound hypersensitivity. The complete questionnaire is displayed in Figure 1.

\section{Hospital Anxiety and Depression Scale}

The HADS was used to screen for excessive depression and anxiety in participants. It is composed of a 14-item questionnaire and evidence of validation has been demonstrated for use in the outpatient setting (22). Seven questions specifically assess the severity of both anxiety and depression. The possible score range is from 0 to 21 for each subscale, with a score of 11 or higher for the subscale suggesting presence of the mood disorder (23).

\section{Uncomfortable Loudness Level}

The protocol for ULL testing in this study is described as follows: the participant sat in a sound booth facing the tester. First, pure tone thresholds at 250, 500, 1000, 2000, 3000, 4000, and $8000 \mathrm{~Hz}$ were obtained. For each tested frequency, a 1 second steady pure tone was presented via insert or headphone in an ascending order starting at 60 decibel $(\mathrm{dB})$ hearing level (HL). The patient reported if the sound was either "ok" or "uncomfortably loud."' When the tone reached the uncomfortably loud level, the step size was decreased to determine the ULL within a $1 \mathrm{~dB}$ resolution. To ensure reliability, the ULL was measured twice at each frequency, and an average of the two presentations was reported. The normal reference level for the ULL is traditionally accepted at $100 \mathrm{~dB}$ HL, although normal hearing individuals have been found to have ULLs between 86 and $98 \mathrm{~dB}$ HL $(24,25)$.

\section{Surgical Technique}

A trans-canal round window and oval window reinforcement under general anesthesia was performed similar to an approach for middle ear procedures. For hemostasis, a four-quadrant 


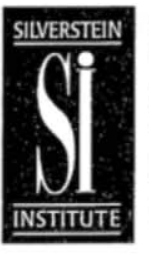

SILVERSTEIN INSTITUTE

Sarasota Office

1901 Floyd Street

Sarasota, FL 34239

Venice Office

400 S. Tamiami Trail, Suite 260

Venice, FL 34285

(941) $366-9222$

Toll Free (888) 418-9200

\section{HYPERACUSIS QUESTIONNAIRE}

\begin{tabular}{|c|c|c|c|c|}
\hline & & & & \\
\hline $\begin{array}{l}{ }^{\star} \text { Please read each item below and } \\
\text { rate it based on the Four-Point Scale. }\end{array}$ & $\begin{array}{c}0 \\
\text { NEVER } \\
\text { Correct }\end{array}$ & $\begin{array}{c}1 \\
\text { SOMETIMES } \\
\text { Correct }\end{array}$ & $\begin{array}{c}2 \\
\text { OFTEN } \\
\text { Correct }\end{array}$ & $\begin{array}{c}3 \\
\text { ALWAYS } \\
\text { Correct }\end{array}$ \\
\hline 1. Sounds that didn't disturb me earlier frighten me now. & 口 & ב & 口 & a \\
\hline $\begin{array}{l}\text { 2. I worry that I will never succeed in getting used to loud/ } \\
\text { uncomfortable sounds. }\end{array}$ & a & a & a & ב \\
\hline $\begin{array}{l}\text { 3. I cannot listen for a long time when I am surrounded by } \\
\text { loud/uncomfortable sounds. }\end{array}$ & ב & a & a & ב \\
\hline $\begin{array}{l}\text { 4. Because of my hypersensitivity to sound, there is tension } \\
\text { between my partner and/or my family and myself. }\end{array}$ & a & a & 口 & a \\
\hline 5. I have to avoid certain sounds. & ב & a & a & a \\
\hline 6. I am very scared of noise. & a & a & $\square$ & ב \\
\hline 7. I think the hypersensitivity to sound has ruined my life. & a & a & 口 & a \\
\hline 8. When surrounded by a lot of sounds, I don't understand anything. & a & a & a & a \\
\hline $\begin{array}{l}\text { 9. Other people distance themselves from me because I can't stand } \\
\text { loud/uncomfortable sounds. }\end{array}$ & a & a & 口 & a \\
\hline 10. I am annoyed by sounds that are too loud/uncomfortable for me. & a & ב & a & a \\
\hline 11. Loud/uncomfortable sounds cause physical pain in my ears. & a & a & 口 & a \\
\hline $\begin{array}{l}\text { 12. I believe I won't be able to cope in everyday life if hypersensitivity } \\
\text { to sound continues to be this bad. }\end{array}$ & a & a & a & a \\
\hline 13. I immediately withdraw when there are loud/uncomfortable sounds. & a & a & 口 & 口 \\
\hline 14. I am afraid that loud/uncomfortable sounds damage my hearing. & a & a & a & a \\
\hline 15. Since becoming hypersensitive to sound, I no longer enjoy music. & a & a & $\square$ & ב \\
\hline Last Name __ & First Name & & & \\
\hline Degree of Incapacity & & & & \\
\hline Slight incapacity & & & & \\
\hline Moderate incapacity & & & & \\
\hline Severe incapacity & & & & \\
\hline Very severe incapacity & & & & \\
\hline
\end{tabular}

FIG. 1. Hyperacusis questionnaire.

injection in the ear canal and tragus was made with $1 \%$ Lidocaine with 1:100,000 Epinephrine. Vertical canal incisions were made at 6 o'clock and 12 o'clock position and a standard tympanomeatal flap was elevated. The middle ear was entered and chorda tympani was preserved in all cases. If additional exposure was needed, the bony posterior external canal was drilled down with a $1.5 \mathrm{~mm}$ diamond burr or curetted to allow adequate visualization of the ossicular chain, round window niche, chorda tympani, facial nerve, and hypotympanum (Fig. 2A). When necessary, additional drilling of the bony round window niche was performed with a $1.0 \mathrm{~mm}$ diamond burr for exposure of the round window membrane. In the first two cases in this series, temporalis fascia graft was used. However, because of incision discomfort, tragal perichondrium graft was used in remaining cases. Through a separate $1 \mathrm{~cm}$ incision, a small piece of the perichondrium was taken leaving the tragal cartilage intact. The graft was flattened with the fascia press; 4 and $2 \mathrm{~mm}$ round grafts were obtained with a biopsy punch. The mucosa of the round window niche and the stapes footplate was scraped with a micro pick to facilitate tissue 

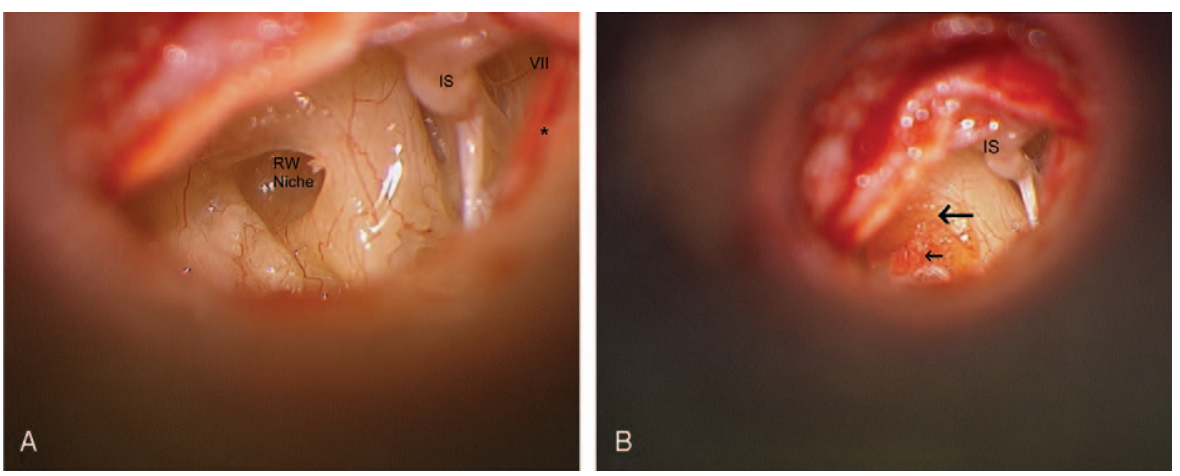

FIG. 2. A, Middle ear exposure. Incudostapedial joint (IS), chorda tympani nerve (*), facial nerve (VII). B, Left ear. Transcanal approach. Tragal perichondrim covering the round window niche (big arrow) and Gelfoam to hold the fascia in place (small arrow). Incudostapedial joint (IS).

welding. The $4 \mathrm{~mm}$ graft was used for the round window and the $2 \mathrm{~mm}$ graft was placed between the stapes crura on the stapes footplate (Fig. 2B). A small piece of Gelfoam was placed over the round window graft to hold it in place against the round window. The tympanomeatal flap was re-approximated along the posterior canal wall and the canal was filled with mupirocin ointment. Fast absorbing suture was used to close tragal incision.

\section{Statistical Analyses}

For analyses, the demographic variables were expressed as means, standard deviation (SD), and the range. Because of the small sample size, parametric analyses were not used. Mean scores with $95 \%$ confidence intervals (CI) were calculated for the outcome variables at each interval visit and plotted to examine the trends of scores over time. Microsoft Excel (Microsoft Corporation, Redmond WA, USA), (2010) was used for all statistical analyses.

\section{RESULTS}

The sample was formed of six subjects: four women and two men whose mean age was $67(\mathrm{SD}=9)$ years, ranging from 55 to 77 years. The sample was further subdivided into two groups depending on whether they underwent unilateral or bilateral reinforcement procedures. The audiometric measures for all participants are displayed in Table 1.

TABLE 1. Audiometric evaluation

\begin{tabular}{lccc}
\hline Subject & $\begin{array}{c}\text { Pre-op } \\
\text { PTA (dB) }\end{array}$ & $\begin{array}{c}\text { 1 Month } \\
\text { Post-op PTA (dB) }\end{array}$ & $\begin{array}{c}\text { 6 Months } \\
\text { Post-op PTA (dB) }\end{array}$ \\
\hline 1 & 5 & 9 & 10 \\
2 & 86 & 86 & 86 \\
3 & 50 & 60 & 55 \\
4A & 26 & 29 & 29 \\
4B & 33 & 38 & 38 \\
5A & 5 & 8 & 8 \\
5B & 6 & 13 & 13 \\
6A & 23 & 33 & 30 \\
6B & 30 & 33 & 36 \\
\hline
\end{tabular}

PTA indicates pure tone average; Post-op, postoperative; Pre-op, preoperative.

\section{Unilateral Group}

This group included three participants: one woman and two men. The mean duration of their hyperacusis was 30 $(\mathrm{SD}=17)$ months, with a range between 24 and 36 months. Two had unknown causes for their hyperacusis and the third developed hyperacusis radiation for an acoustic neuroma radiation. Temporalis fascia was used for the round window reinforcement in two participants, while tragal perichondrium was used for one participant. After the treatment, all the participants reported subjective improvement as evidenced by an improved mean score of $28(\mathrm{SD}=14)$ on the HQ (Fig. 3A). The mean ULL value at baseline was $77 \mathrm{~dB}(\mathrm{CI}, \pm 12 \mathrm{~dB})$ and at 1 month and 6 months postoperative was $94 \mathrm{~dB}$ (CI, $\pm 10 \mathrm{~dB})$ and $91 \mathrm{~dB}(\mathrm{CI}, \pm 18 \mathrm{~dB})$, respectively (Fig. 3B). These results indicate an improved postoperative mean ULL score of $14 \mathrm{~dB}$ in this group. The patients reported no change in hearing and improved quality of life after the procedure.

\section{Bilateral Group}

In this group, there were three women subjects, totaling six operated ears. In this sample, the mean hyperacusis duration ranged between 18 and 61 months, with a mean of $38(\mathrm{SD}=17)$ months. All had unknown causes for their hyperacusis. Four operated ears had temporalis fascia for the round window reinforcement while two ears were reinforced with tragal perichondrium. Analysis of the ULL measures revealed a mean ULL of $69 \mathrm{~dB}$ (CI, $\pm 5 \mathrm{~dB})$ at baseline for the first ear. After 1 month, the mean ULL values were $81 \mathrm{~dB}(\mathrm{CI}, \pm 9 \mathrm{~dB})$ and $82 \mathrm{~dB}(\mathrm{CI}$, $\pm 15 \mathrm{~dB})$ at 6 months postoperatively. Mean ULL values were $75 \mathrm{~dB}(\mathrm{CI}, \pm 2 \mathrm{~dB})$ pre-op for the second ear and at 1 and 6 months after the procedure were $85 \mathrm{~dB}(\mathrm{CI}, \pm 5 \mathrm{~dB})$ and $83 \mathrm{~dB}(\mathrm{CI}, \pm 11 \mathrm{~dB})$ respectively (Fig. $4, \mathrm{~A}$ and $\mathrm{B}$ ). After the treatment, two of the three participants reported subjective improvement in their sound hypersensitivity. The mean HQ score was $29.6(\mathrm{SD}=6)$ preoperatively and 16.86 months after surgery (Fig. 5). One patient underwent revision surgery on the first operated ear to place more fascia because of her lack of improvement on ULL testing. There was minimal improvement noted in 

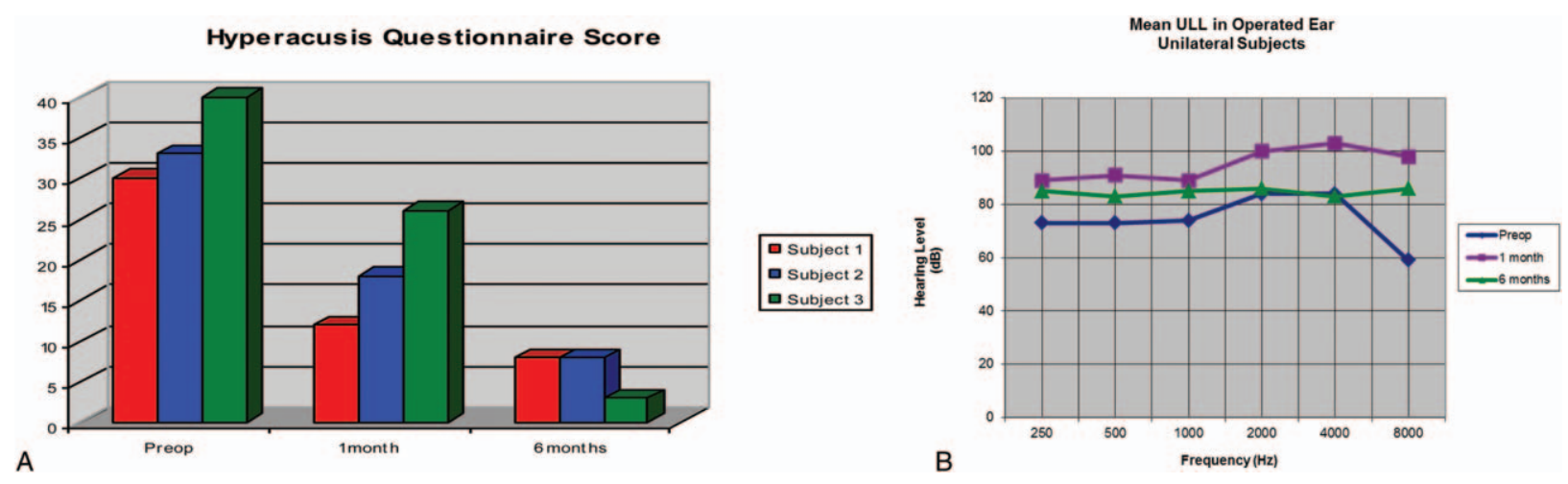

FIG. 3. A, Hyperacusis questionnaire improvement unilateral subjects. $B$, Uncomfortable loudness level (ULL) unilateral subjects.
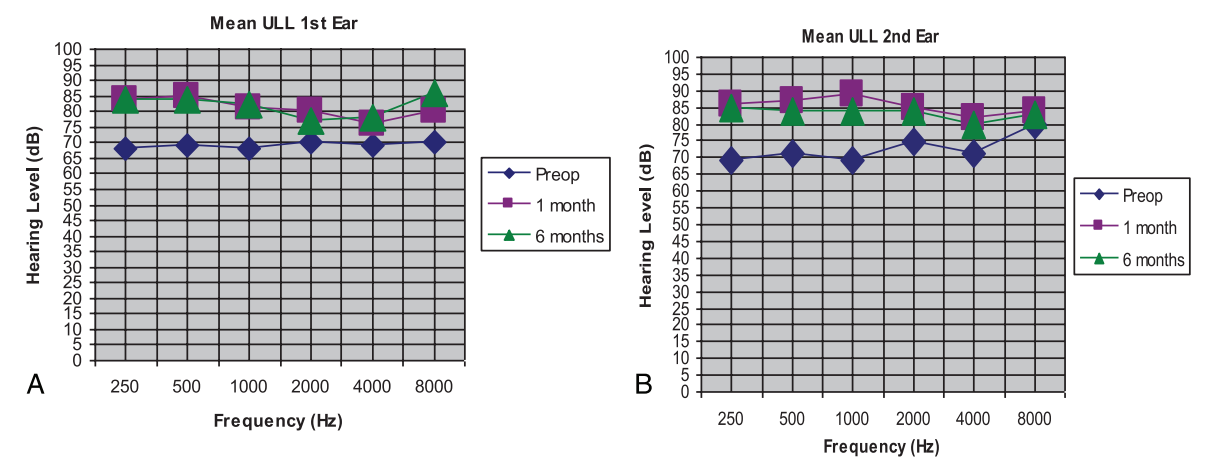

FIG. 4. A, Uncomfortable loudness level (ULL) bilateral subjects_-1st ear. B, Uncomfortable loudness level (ULL) bilateral subjects-2nd ear.

both the ULL testing and HQ score after the revision for this participant. However, the participant stated their noise tolerance improved in social situations. Similar to the subjects in the unilateral group, none had subjective change in their hearing as a result of the procedure and there were no surgical complications. Furthermore, the participants stated their overall quality of life was improved after their procedure(s).

\section{DISCUSSION}

In 2014, Silverstein et al. (20) first reported marked improvement in sound intolerance in two patients after round and oval window reinforcement with temporalis fascia. The present results of this exploratory study suggest that round and oval window reinforcement may offer relief for individuals afflicted with hyperacusis. There was improvement in the postoperative ULL's in both the unilateral and bilateral groups, suggesting improved sound tolerance. Most patients noted their greatest improvement after the first ear surgery. Further, subjective improvement was observed after the second ear surgery despite minimal improvement in psychoacoustic measures. While the second ear surgery does not show a significant increase in ULL, the participants' subjective statements substantiate the benefit of second ear surgery. Similar phenomena where greatest benefit occurs after the first ear surgery has been observed in subjects who underwent bilateral stapedotomies (26). While we were not able to perform statistical testing because of our small sample size, a positive trend was noted with improvement of the ULLs when analyzing first ear and second ear surgeries.

Hyperacusis has been generally accepted to be related to an increase in auditory responsiveness arising from auditory neuronal degeneration. Previously published trials have used a variety of measures and protocols for the assessment of hyperacusis with variable results $(16,27)$. Moreover, there is significant variability in the interpretation of the test data recommended for the

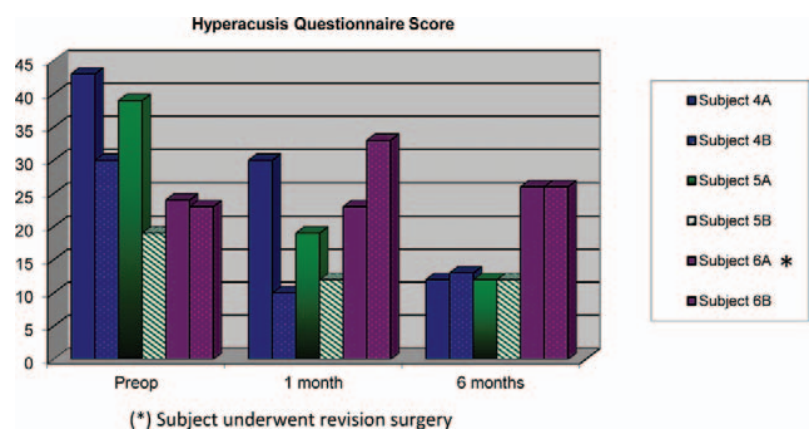

FIG. 5. Hyperacusis questionnaire improvement bilateral subjects. 
identification of hyperacusis. ULLs are the most frequently used in the assessment of hyperacusis (28). The ULL is a commonly used test in the hearing aid fitting process (29). Recent studies have shown ULL to complement the hearing evaluation in patients with noise intolerance (30). This testing can determine the minimum level of sounds that is judged to be uncomfortably loud by the patient. The terms loudness discomfort level and ULL are used interchangeably among investigators in this specialty area. The accepted normal reference level for the ULL is considered $100 \mathrm{~dB} \mathrm{HL}$ for the frequencies from 500 to $8000 \mathrm{~Hz}$ and for speech according to American Academy of Audiology (31). However, caution must be exercised when interpreting ULL measures as they may lack the specificity and sensitivity necessary to confirm a diagnosis of hyperacusis (7). Test-retest reliability has also been questioned. ULL scores are strongly dependent on the consistency and accuracy of the instructions provided by the tester. However, other studies have suggested the ULL is a good instrument for the assessment and follow-up of patients with sound intolerance. Therefore, the utility of ULLs is mixed $(1,32)$. Examination of HQ data showed scores were improved postoperatively in five out of six subjects. In the unilateral group, HQ scores were substantially decreased at 1 month and continued to decline at 6 months. However, the most significant improvement in HQ scores was observed in bilateral subjects at 1 month after first and second ear surgery, but scores appeared to plateau at 6 months. A definitive explanation for this observation is elusive at this time because of the small sample and the addition of the second ear surgery at only 1 month after the first ear procedure. The effect of these confounding variables needs to be further explored. Interestingly, item analysis of the HQ revealed a reduction in scores for the somatic behavior or reaction linked to specific situation subscale in all subjects. Because of the lack of availability of subjective measures for hyperacusis in English with evidence for validity and reliability, a non-validated questionnaire was used. Nevertheless, these observations reinforce that the dampening procedure may have rendered the inner ear less sensitive to the effect of sudden changes in sound and pressure, therefore decreasing the hypersensitivity of the inner ear. This was further supported by subject accounts of resuming activities postoperatively including dining out, social interaction and they reported improved interpersonal relationships, specifically with their spouse. Most frequently, misophonia may accompany hyperacusis and it is important to assess the extent of misophonia. Because of the mechanism of misophonia and its functional link to the limbic system, this surgery is not helpful for these patient populations.

As stated previously, while statistical inferences cannot be made because of the small sample size of this exploratory study, the results of both the psychoacoustic testing and the subjective measures suggest this minimally invasive procedure offers significant clinical implications that warrant further investigation.
This study has several limitations. Because of the small sample size of our cohort, sample variability was increased and unfortunately we lacked statistical power. The use of a non-validated self-report questionnaire limits interpretation of the participants' HQ scores in regards to sound hypersensitivity. In future studies, we may consider the Multiple-Activity Scale for Hyperacusis (MASH) as a tool to measure an individual's level of annoyance in relation to hypersensitivity to sound (33) as well as the tinnitus handicap inventory to assess tinnitus improvement over time after the procedure. Performing the second ear surgery at 1 month made the ULL data from the un-operated ear more difficult to interpret and we were unable to control this variable when collecting data for the initial ear that was treated. In future studies, we plan to wait a full 6 months before performing the procedure on the second ear to allow for full data collection on each ear, thus enhancing the understanding of the potential benefit of the unilateral versus bilateral procedure. Another potential limitation is the possibility of a placebo effect in the treated subjects. When present, a placebo effect threatens a study's internal validity. In the present study, the outcome measures used to assess the efficacy of the round and oval window reinforcement procedure were subjective. Therefore, response bias, the notion that a participant offered positive responses to the questionnaires just because they received an intervention or because they wanted to please the researcher must be considered. The use of a control group with no intervention, and the addition of objective measures in future studies may be helpful to determine the true effect of this novel minimally invasive procedure.

Our results offer supportive evidence that when the ULL is below $90 \mathrm{~dB}$, minimally invasive surgery using reinforcement of the round and oval window with either temporalis fascia or tragal perichondrium may reduce noise sensitivity in individuals with intractable hyperacusis. The participants of this exploratory study reported diminished sound intolerance and improved quality of life. In patients with bilateral hyperacusis, there appears to be a greater improvement with noise tolerance after the first surgery. The second ear surgery added minimal benefit as evidenced by the ULL results. However, the participants stated they experienced decreased sound sensitivity after the second procedure. Further investigation of this innovative technique is needed.

\section{REFERENCES}

1. Baguley D, Andersson G. Hyperacusis: Mechanism, diagnosis, and therapies. Plural; 2007.

2. Blaesing L, Kroener-Herwig B. Self-reported and behavioral sound avoidance in tinnitus and hyperacusis subjects, and association with anxiety ratings. Int $J$ Audiol 2012;51:5111-7.

3. Fackrell K, Fearnley C, Hoare DJ, Sereda M. Hyperacusis questionnaire as a tool for measuring hypersensitivity to sound in a tinnitus research population. Biomed Res Int 2015;2015:1-12.

4. Hannula S, Bloigu R, Majamaa K, Sorri M, Mäki-Torkko E. Selfreported hearing problems among older adults: prevalence and comparison to measured hearing impairment. $J$ Am Acad Audiol 2011;22:550-9. 
5. Andersson G, Lindvall N, Hursti T, Carlbring P. Hypersensitivity to sound (hyperacusis): a prevalence study conducted via the internet and post. Int J Audiol 2002;41:545-54.

6. Jastreboff MM, Jastreboof PJ. Decreased sound tolerance and tinnitus retraining therapy (TRT). Aust $N Z J$ Audiol 2002; $24: 74-81$.

7. Sheldrake J, Diehl P, Schaette R. Audiometric characteristics of hyperacusis patients. Front Neurol 2015;6:105.

8. Jastreboff PJ, Jastreboff MM. Tinnitus retraining therapy for patients with tinnitus and decreased sound tolerance. Otolaryngol Clin North Am 2003;36:321-36.

9. Henry JA, Jastreboff MM, Jastreboff PJ, et al. Assessment of patients for treatment with tinnitus retraining therapy. $J$ Am Acad Audiol 2002;13:523-44.

10. Herbert S, Fournier P, Norena A. The auditory sensitivity is increased in tinnitus ears. J Neurosci 2013;33:2356-64.

11. Vernon J. Recruitment. When noise spells pain. The Voice 1991;12:37.

12. Knipper M, Van Dijk P, Nunes I, Rüttiger L, Zimmermann U. Advances in the neurobiology of hearing disorders: recent developments regarding the basis of tinnitus and hyperacusis. Prog Neurobiol 2013;111:17-33.

13. Fukaya T, Nomura Y. Audiological aspects of idiopathic perilymphatic fistula. Acta Otolaryngol Suppl 1988;456:68-73.

14. Nelting M, Rienhoff NK, Hesse G, Lamparter U. The assessment of subjective distress related to hyperacusis with a self-rating questionnaire on hypersensitivity to sound. [In German]. Laryngorhinootologie 2002;81:327-34.

15. Aazh H, McFerran D, Salvi R, Prasher D, Jastreboff M, Jastreboff P. Insights from the first international conference on hyperacusis: causes, evaluation, diagnosis and treatment. Noise Health 2014; $16: 123-6$.

16. Juris L, Andersson G, Larsen HC, Ekselius L. Cognitive behaviour therapy for hypercausis: a randomized controlled trial. Behav Res Ther 2014;54:30-7.

17. Silverstein H, Kartush JM, Parnes LS, et al. Round window reinforcement for superior semicircular canal dehiscence: a retrospective multi-center case series. Am J Otolaryngol 2014;3:286-93.

18. Nikkar-Esfahani A, Whelan D, Banerjee A. A occlusion of the round window: a novel way to treat hyperacusis symptoms in superior semicircular canal dehiscence syndrome. J Laryngol Otol 2013;127:705-7.

19. Dang PT, Kennedy TA, Gubbels SP. Simultaneous, unilateral plugging of superior and posterior semicircular canal dehiscences to treat debilitating hyperacusis. J Laryngol Otol 2014;128:174-8.
20. Silverstein H, Wu YH, Hagan S. Round and oval window reinforcement for the treatment of hyperacusis. Am $J$ Otolaryngol 2014;26:158-62.

21. Khalfa S, Dubal S, Veuillet E, Perez-Diaz F, Jouvent R, Collet L. Psychometric normalization of a hyperacusis questionnaire. ORL $J$ Otorhinolaryngol Relat Spec 2002;64:436-42.

22. Bjelland L, Dahl AA, Haug TT, Neckelmann D. The validity of the hospital anxiety and depression scale. An updated literature review. J Psychosom Res 2002;52:69-77.

23. Zigmond AS, Snaith RP. The hospital anxiety and depression scale. Acta Psychiatr Scand 1983;67:361-70.

24. Wallen MB, Hasson D, Theorell T, Canlon B. The correlation between the hyperacusis questionnaire and uncomfortable loudness levels is dependent on emotional exhaustion. Int $J$ Audiol 2012;51:722-9.

25. Goldstein B, Shulman A. Tinnitus-hyperacusis and the loudness discomfort level test-a preliminary report. Int Tinnitus $J$ 1996;2:83-9.

26. Kisilevsky VE, Bailie NA, Dutt SN, Halik JJ. Functional results of 394 bilateral stapedotomies evaluated with the Glasgow benefit plot. Eur Arch Otorhinolaryngol 2010;267:1027-34.

27. Pienkowski M, Tyler RS, Roncancio ER, et al. A review of hyperacusis and future directions:part II. Measurement, mechanisms, and treatment. Am J Audiol 2014;23:420-36.

28. Hazell JW, Sheldrake JB. Hyperacusis and tinnitus. In: Aran J-M, Dauman R, editors. Proceedings IV International Tinnitus Seminar, Bordeaux 1991. Amsterdam: Kugler, Gbedini Publications; 1992. pp. $245-8$.

29. Bentler RA, Cooley LJ. An examination of several characteristics that affect the prediction of OSPL90 in hearing aids. Ear Hear 2001;1:58-64.

30. Jastreboff PJ, Jastreboff MM. Decreased sound tolerance. In: Snow Jr J, editor. Tinnitus: Theory and Management. London, Hamilton: BC Decker; 2004. pp. 8-15. Ch. 2.

31. Katzenell U, Segal S. Hyperacusis: review and clinical guidelines. Oto Neurotol 2001;22:321-6.

32. Dawson J. A comparison of physical measurements of pure-tones, third-octave bands of noise and third-octave bands of speech to subjective judgments of audibility threshold, MCL and UCL for three normally hearing listeners (Independent Studies and Capstones, Paper No. 306). St. Louis, MO: Washington University School of Medicine, Program in Audiology and Communication Sciences.

33. Dauman R, Bouscau-Faure F. Assessment and amelioration of hyperacusis in tinnitus patients. Acta Otolaryngologica 2005;125:503-9. 